\title{
Design and Implementation of a Checklist for Prediction of Anesthesia-Induced Nausea and Vomiting in Candidate Patients for Mastectomy
}

\author{
Mehdi Khanbabayi Gol ${ }^{\circledR}$, Maryam Dadashzadeh ${ }^{2}$, Hassan Mohammadipour Anvari ${ }^{3^{*}(\mathbb{D}}$
}

\begin{abstract}
Objectives: Prediction of nausea and vomiting can positively contribute to the management of this post-anesthesia adverse effect. Therefore, the present study aimed to design and implement a checklist for predicting anesthesia-induced nausea and vomiting in candidate patients for mastectomy.

Materials and Methods: This methodological study was conducted on 300 candidate patients for mastectomy during 2018-2019 at Imam Reza hospital, Tabriz, Iran. The checklist items were designed and developed based on scientific articles, expert opinions, and patient interviews. The Pearson correlation coefficient, Cronbach's alpha, Spearman-Brown coefficient, factor analysis, the KaiserMeyer-Olsen measure of sampling adequacy, and VARIMAX rotation were used to analyze the data. Eventually, the distribution of data with a normal distribution was compared through the Kolmogorov-Smirnov test.

Results: In the first stage, 100 items were collected, which were reduced to 35 cases after modification by a team of experts. Twenty items were ultimately selected after observing the $80 / 20$ response rate. The overall scale reliability was calculated as 0.953 based on Cronbach's alpha. The correlation coefficient of the first and second implementations was 0.853 . Finally, the four extracted factors accounted for 69.51 of the variance of the checklist based on factor analysis.

Conclusions: The prediction checklist for post-anesthesia nausea and vomiting in candidate patients for mastectomy comprised adequate psychometric indicators that could be used with high reliability according to the extracted indices.

Keywords: Test design, Standardization, Prediction of nausea and vomiting, Mastectomy, Anesthesia
\end{abstract}

\section{Introduction}

Nausea and vomiting are the second most adverse and problematic postoperative complications with an incidence of $20 \%-30 \%$ (1). In addition, they represent an unpleasant experience for patients who identify them as being worse than postoperative pain. The prevention of postoperative nausea and vomiting in patients at high risk of these complications improves patient satisfaction. However, it reduces the incidence of postoperative complications, prevents recovery room discharge delays and unanticipated prolonged hospital stays following a surgery, and ultimately, reduces postoperative nauseaand vomiting-induced health care costs $(2,3)$.

Breast cancer treatments include chemotherapy, surgery, biotherapy, and radiotherapy, among which surgery is performed on a large number of patients (4). Numerous factors are identified to cause post-mastectomy nausea in anesthetized patients. Given the multiplicity of these factors, predicting this complication is difficult for anesthetists and hence its management for anesthetists and other healthcare providers is a complicated issue (5). Considering the direct influence of post-mastectomy nausea and vomiting prediction on the surgery outcome, the need for medication, and management of this complication, collecting all contributing factors in the form of a checklist that can be used as a prediction instrument can facilitate the tasks of medical practitioners. Accordingly, the present study aimed to design and implement a checklist for predicting anesthesia-induced nausea and vomiting in candidate patients for mastectomy.

\section{Materials and Methods}

This was a methodological study that led to the development of an instrument and was conducted from December 1, 2018, to April 19, 2019, at Imam Reza Hospital, Tabriz, Iran. A sample size of 300 was considered in the present study since the most suitable samples should be over 300 individuals for factor analysis and 300 for normal groups $(6,7)$. The inclusion criteria were breast cancer, the minimum education of high school diploma, and needing mastectomy and the exclusion criteria encompassed the lack of consent to participate in the study and psychosis.

The first phase of instrument development included searching credible scientific articles for determining the contributing factors to postoperative nausea and vomiting. The resulting checklist was completed through 
conducting interviews with the participants on the contributing factors to nausea and vomiting. The expert opinions (from 5 professors of anesthesia and instrument development) were subsequently sought to modify and put the contributing factors in correct wording.

All ethical principles for research conduction and the requirements, adhered to in the majority of studies (814), were observed by the researchers. These principles included obtaining the code of ethics (IR.TBZMED. REC.1397.598) and written informed consent from participants, introducing the researchers to research departments, observing voluntary participation in the study, utilizing the study results in case of patient need, and obtaining permission to record participants' voices.

\section{Data Analysis}

The test-retest reliability coefficient and the Pearson correlation coefficient were used to determine construct validity and the parallel form reliability, respectively. The internal consistency reliability was determined through Cronbach's alpha and the Spearman-Brown coefficient. In addition, factor analysis, the Kaiser-Meyer-Olsen (KMO) measure of sampling adequacy, and VARIMAX rotation were used to determine construct validity. Finally, the distribution of data with a normal distribution was compared through the Kolmogorov-Smirnov test.

\section{Results}

The study results are presented in 4 parts including the selection of scale items, scale validity, scale reliability, and cut-off point determination.

\section{Initial Selection of Scale Items}

As described in the instrumentation section, items from various scientific sources were extracted and combined with the materials collected in the interviews. The initial checklist consisted of 100 items which were reduced to 35 after examination by the experts. In the pilot implementation, 2 indicators were regarded as the basis for removing or selecting the items, including observing the $80 / 20$ response rate and the interval between the 2 values, as well as the Cronbach's alpha value and the contribution of each item to the reduction or increase of this index. Based on the $80 / 20$ criterion, items selected by less than $20 \%$ or more than $80 \%$ of individuals should be removed or modified owing to the minor variation created by them and the more inadequate identification of individual differences. The number of such responses was limited in this study. Given that this scale is classified under attitude assessment scales, the $80 / 20$ criterion is overlooked when possible since it is primarily applied in developing cognitive and developmental assessment scales. This point was also discussed with the experts. In addition, a number of the contributing factors to nausea and vomiting causing severe psychological burden were occasionally selected by $90 \%$ of the patients. Therefore, theoretical studies also prevented the use of this method regarding eliminating the items. At this stage, 15 items were removed due to the similarity of content with other items and the resulting similar response pattern. The target scale was then implemented with 20 items on the target sample. No item was removed at this stage based on the contribution of each item to the Cronbach's alpha value.

\section{Validity Analysis}

Content Validity

After the specified procedure, the 20-item scale was distributed among the experts for the final review. All experts expressed their agreement on the predictive nature of items for post-anesthetic nausea and vomiting, pointing to the adequate content validity of the checklist.

\section{Construct Validity}

The accepted principle in construct validity indicates that if a scale is to measure the intended attribute according to expectations, it must be positively correlated with the consistent constructs while negatively correlated with inconsistent constructs (Table 1).

The General Health Questionnaire (GHQ) was used to assess divergent validity (the 28 -item form). Thirty people in the elderly group filled out GHQ. In addition to completing the prediction checklist for nausea and vomiting. The correlation coefficient of the 2 scales is as follows.

As shown, all coefficients are statistically significant with a confidence level of 0.99 , demonstrating that the prediction of the increased incidence of nausea and vomiting increases the probability of harm from any of the above-mentioned subscales (i.e., decreased health), which indicates the construct validity of the scale.

Other methods of estimating the scale validity include factor analysis and the reduction of items to fewer and more significant variables. After establishing the veracity of the assumptions of factor analysis, the resulting data were analyzed using principal component analysis. Four factors were subsequently extracted as follows, accounting for 69.51 of the variance of the checklist (Table 2).

Table 2 lists the eigenvalues for scale items. An eigenvalue is the degree of the variance of the items based on a factor. In other words, it is a value of the total test

Table 1. The Observed Coefficient of the Prediction Checklist for Anesthesia-induced Nausea and Vomiting in Mastectomy Candidate Patients and GHQ Subscales

\begin{tabular}{lc}
\hline GHQ & Prediction Checklist for Nausea and Vomiting \\
\hline Psychophysical problems & -0.31 \\
Social function disorder & -0.39 \\
Sleep anxiety and disorder & -0.46 \\
Depression & -0.40 \\
\hline
\end{tabular}

Note. GHQ, General health questionnaire. 
Table 2. Variance Values Before and After Rotation and Eigenvalues

\begin{tabular}{|c|c|c|c|c|c|c|c|c|}
\hline \multicolumn{3}{|c|}{ Results After Rotation } & \multicolumn{3}{|c|}{ Results Before Rotation } & \multicolumn{3}{|c|}{ Initial Eigenvalue } \\
\hline $\begin{array}{l}\text { Cumulative } \\
\text { Percentage }\end{array}$ & $\begin{array}{l}\text { Variance } \\
\text { Percentage }\end{array}$ & Total & $\begin{array}{l}\text { Cumulative } \\
\text { Percentage }\end{array}$ & $\begin{array}{l}\text { Variance } \\
\text { Percentage }\end{array}$ & Total & $\begin{array}{l}\text { Cumulative } \\
\text { Percentage }\end{array}$ & $\begin{array}{l}\text { Variance } \\
\text { Percentage }\end{array}$ & Total \\
\hline 16.491 & 15.192 & 6.177 & 28.511 & 27.459 & 11.119 & 6.177 & 15.192 & 16.491 \\
\hline 31.512 & 15.391 & 6.129 & 40.500 & 13.039 & 5.291 & 6.129 & 15.391 & 31.512 \\
\hline 55.693 & 11.311 & 4.452 & 50.199 & 10.115 & 4.811 & 4.452 & 11.311 & 55.693 \\
\hline 69.512 & 2.758 & 1.109 & 69.512 & 2.711 & 1.012 & 1.109 & 2.758 & 69.512 \\
\hline
\end{tabular}

variance that is estimated by a particular factor and is calculated through dividing the eigenvalue of each factor by the number of items. The fourth column indicates the cumulative variance. Further, the fifth, sixth, and seventh columns demonstrate the pre-rotation eigenvalues, the percentage of the explained variance, and the percentage of the cumulative variance for items with an eigenvalue greater than or equal to one, respectively. The eighth, ninth, and tenth columns represent the same indicators after rotation as well.

\section{Selection of Factors}

The KMO and the Cattell's scree test were used to select the appropriate factors. The number of factors with eigenvalues equal to or greater than one is considered as the limit in the KMO. Table 3 presents the postrotation factor loadings of the items from the extracted components.

The specified scale was once again investigated and discussed by the group of experts in order to designate the extracted factors. This stage of scale development is

Table 3. Factor Matrices of the Four Extracted Factors Using an EQUAMAX Rotation

\begin{tabular}{lcccc}
\hline \multirow{2}{*}{ Items } & \multicolumn{4}{c}{ Factors } \\
\cline { 2 - 4 } & First factor & Second factor & Third factor & Fourth factor \\
\hline 1 & 0.781 & & & \\
2 & & 0.531 & & \\
3 & & 0.801 & \\
4 & 0.851 & & & 0.612 \\
5 & 0.725 & & & 0.913 \\
6 & & & & \\
7 & & & & \\
8 & & 0.911 & 0.601 & \\
9 & & 0.721 & 0.789 & \\
10 & & & 0.693 & \\
11 & & & 0.679 & \\
12 & & & & \\
13 & & & & \\
14 & & & & \\
15 & 0.903 & & & \\
16 & & & & \\
17 & & & & \\
18 & & & & \\
19 & & & & \\
20 & & & & \\
\hline
\end{tabular}

another testament to its content validity. Designating the extracted factors requires reviewing their content and selecting the appropriate titles accordingly. The 4 factors were agreed to be designated as administered medications, comorbidities, respiratory status, and electrolyte status.

\section{Scale Reliability}

Internal consistency, split-half reliability, the test-retest reliability coefficient, and the parallel form reliability were used to estimate the test reliability.

\section{Internal Consistency Method}

The internal consistency of the checklist was estimated using Cronbach's alpha and the results of the analysis showed that the overall alpha value of the scale was 0.953 , which is shown for each component in Table 4.

\section{Estimation of Split-half Reliability}

The correlation coefficient of the 2 splits of the test indicates its reliability. The estimation of the reliability based on the Spearman split-half coefficient was 0.73. Furthermore, this value was 0.911 for the overall scale after correction and calculation of the Spearman-Brown coefficient.

To achieve the test-retest reliability, the scale was reimplemented on 30 candidate patients for a mastectomy after 2 weeks of initial implementation. According to the retest results, the correlation coefficient of the first and second implementations was 0.853 , representing the test reliability.

\section{Determination of the Scale Cut-off Point}

The cut-off points were extracted using the confidence intervals, mean, and standard deviation. The results of the Kolmogorov-Smirnov test revealed no significant difference between the observed and normal distribution scores. Therefore, the confidence interval index can be used to arrive at the cut-off points given the presence of the normal distribution characteristics for the existing scores (Table 5).

As shown in Table 6, those candidate patients with a score of less than 3 on the prediction checklist for postanesthesia nausea and vomiting for mastectomy are very unlikely to develop post-anesthesia nausea and vomiting. In addition, the prediction scores of 4-7, 8-11, 12-16, and 
Table 4. Reliability Coefficients by Factors

\begin{tabular}{lc}
\hline Subscales & Reliability Coefficient \\
\hline Administered medications & 0.978 \\
Comorbidities & 0.935 \\
Respiratory status & 0.941 \\
Electrolyte status & 0.961 \\
\hline
\end{tabular}

Note. SD: Standard deviation.

Table 5. Comparison of the Observed and Normal Distribution Scores

\begin{tabular}{llc}
\hline & & Total Score \\
\hline Sample size & & 300 \\
\hline \multirow{2}{*}{ Normal parameters } & Mean & 18.274 \\
\cline { 2 - 3 } & SD & 5.11 \\
\hline \multirow{2}{*}{ Maximum difference } & Definitive & 0.40 \\
\cline { 2 - 3 } & Positive & 0.40 \\
\cline { 2 - 3 } & Negative & -0.39 \\
\hline Kolmogorov-Smirnov test value & & 0.721 \\
\hline Significance level & & 0.626 \\
\hline
\end{tabular}

Note. SD: Standard deviation.

Table 6. Checklist Points Predict Nausea and Vomiting After Anesthesia

\begin{tabular}{lc}
\hline Severity of Nausea & Cut-off Point \\
\hline Extremely severe & $17-20$ \\
Severe & $12-16$ \\
Average & $8-11$ \\
Mild & $4-7$ \\
Extremely mild & $0-3$ \\
\hline
\end{tabular}

$\geq 17$ demonstrate a low, average, high, and extremely high probability of developing nausea, respectively (Table 6).

\section{Discussion}

The possible methods were used to examine the psychometric indicators of the prediction checklist for post-anesthesia nausea and vomiting in candidate patients for mastectomy (15-17). Evidence regarding the test validity in terms of content validity indicated that the instrument measures the defined attribute correctly. As regards construct validity, the obtained coefficients confirmed the theoretical expectation of the existence of an inverse correlation between inconsistent constructs, indicating that the scale correctly measured the relevant construct and the coefficient of the test validity index. The result of factor analysis highlighted 4 factors that reduce the variables to smaller ones based on the underlying correlation between them. The observation of the content structure of factors demonstrated that each factor consists of an underlying variable that can theoretically cover and predict a number of the contributing factors to nausea and vomiting. Moreover, the expected items in each factor were based on the development of a unit concept of test validity and all factors were designated by a team of experts.

The reliability of the questionnaire was examined in 2 aspects. The test-retest reliability coefficient was calculated as 0.853 , indicating an adequate coefficient and reliable use of the test in different situations. Additionally, the Cronbach's alpha was computed as 0.953 , demonstrating that the items enjoy adequate internal consistency. Other measures such as the split-half reliability point to a well-developed test. The utilized indicators are the most common and reliable methods for estimating the reliability of a test, which are collectively used to confirm the reliability of the test.

The cut-off points were also selected based on the standard deviations of 1.64 and 1.96. In this implementation and according to the cut-off point, $1.7 \%, 15.1 \%, 66.1 \%$, $14.4 \%$, and $2.7 \%$ of people experienced extremely severe, severe, moderate, mild, and extremely mild nausea and vomiting, respectively. The observed distribution borders closely on the theoretical normal distribution, showing no statistically significant difference between the 2 groups. This represents the normal distribution of the attribute throughout the community and serves as an indicator for confirming the cut-off points of the scale.

\section{Conclusions}

The post-anesthesia prediction checklist for nausea and vomiting in candidate patients for mastectomy consists of appropriate psychometric indicators that can be used with great reliability based on the extracted indices. The specified items in the scale were selected and statistically analyzed based on the contributing factors to post-anesthesia nausea and vomiting. The frequency of mastectomy is ever-increasingly rising due to the incidence of cancer, therefore, the existing scale can be used reliably in various research situations. The extracted validity and reliability indices point to a reliable use of the scale. This scale has a cut-off point for distinguishing individuals based on the degree of contributing factors to nausea and vomiting, which can be useful to researchers in prediction studies. Other required statistical data, especially the normal scores are available to interested parties through correspondence with the corresponding author of the article.

\section{Conflict of Interests}

Authors declare that they have no conflict of interests.

\section{Financial Support}

The Research Center and the Health Vice-chancellor of Tabriz University of Medical Sciences.

\section{Acknowledgments}

The present paper presented the results of a part of the research project approved by the Clinical Research Development Unit, Shohada hospital of Tabriz University of Medical Sciences with the ethics code of IR.TBZMED. REC.1397.598. The researchers would like to give their gratitude to the Research Center and the Health Vice- 
chancellor of Tabriz University of Medical Sciences for financial support in the study. It should be noted that this article was derived from the research project and approved by the Tuberculosis and Pulmonary Diseases Research Center.

\section{References}

1. Ghanei Gheshlagh R, Dastras M, Fazlali PourMiyandoab $\mathrm{M}$, Naseri $\mathrm{O}$. The relationship between preoperative anxiety and postoperative nausea and vomiting. Medical Science Journal of Islamic Azad Univesity-Tehran Medical Branch. 2014;23(4):269-274. [Persian].

2. Collins AS. Clinical utility of antiemetics and complementary therapies in the prevention of postoperative nausea and vomiting. Clin Audit. 2013;5:67-76. doi:10.2147/CA.S30588

3. Yeganeh Z, Sheikhan Z, Kariman N, Hajian P, Nasiri M, Mirzadeh N. Relationship between pregnancy-associated variables and breast cancer risk: A systematic review. Iranian Journal of Obstetrics, Gynecology and Infertility. 2018;21(2):85-97. doi:10.22038/ijogi.2018.10716

4. Ganz PA, Kwan L, Stanton AL, Bower JE, Belin TR. Physical and psychosocial recovery in the year after primary treatment of breast cancer. J Clin Oncol. 2011;29(9):11011109. doi:10.1200/jco.2010.28.8043

5. Sabzalizadeh S, Ravaghi H, Salehi M, Delshad V. The effectiveness Comparison of palonosetron with ondansetron in preventing postoperative nausea and vomiting in patient undergoing general anesthesia: A Metaanalysis and Systematic review. Journal of Anesthesiology and Pain. 2016;6(4):66-76. [Persian].

6. Soleimani S, Mofrad F, Kareshki H. Interpersonal Emotion Regulation Questionnaire (IERQ) in Persian speaking population: Adaption, Factor Structure and Psychometric Properties. Int J Appl Behav Sci. 2016;3(4):41-49. doi:10.22037/ijabs.v3i4.16483

7. MacCallum RC, Widaman KF, Zhang S, Hong S. Sample size in factor analysis. Psychol Methods. 1999;4(1):84-99. doi:10.1037\%2F1082-989X.4.1.84

8. Zomorrodi A, Mohammadipoor Anvari H, Kakaei F, Solymanzadeh F, Khanlari E, Bagheri A. Bolus Injection
Versus Infusion of Furosemide in Kidney Transplantation: A Randomized Clinical Trial. Urol J. 2017;14(2):3013-3017.

9. Movassaghi R, Peirovifar A, Aghamohammadi D, Mohammadipour Anvari H, Golzari SE, Kourehpaz Z. Premedication with single dose of acetazolamide for the control of referral shoulder pain after laparoscopic cholecystectomy. Anesth Pain Med. 2015;5(6):e29366. doi:10.5812/aapm.29366

10. Naderpour M, Moghadam YJ, Ghanbarpour E, Shahidi $\mathrm{N}$. Evaluation of factors affecting the surgical outcome in tympanoplasty. Iran J Otorhinolaryngol. 2016;28(85):99104.

11. Fakhim SA, Shahidi N, Lotfi A. Prevalence of associated anomalies in cleft lip and/or palate patients. Iran J Otorhinolaryngol. 2016;28(85):135-9.

12. Naderpour M, Shahidi N, Hemmatjoo T. Comparison of tympanoplasty results in dry and wet ears. Iran J Otorhinolaryngol. 2016;28(86):209-14.

13. Vahedi P, Salehpour F, Aghamohammadi D, et al. Single dose preemptive amitriptyline reduces postoperative neuropathic pain after lumbar laminectomy and discectomy: a randomized placebo-controlled clinical trial. Neurosurg Q. 2010;20(3):151-158. doi:10.1097/ WNQ.0b013e3181ebce15

14. Fakhari S, Bavil FM, Bilehjani E, Abolhasani S, Mirinazhad M, Naghipour B. Prophylactic furosemide infusion decreasing early major postoperative renal dysfunction in on-pump adult cardiac surgery: a randomized clinical trial. Res Rep Urol. 2017;9:5-13. doi:10.2147/rru.s126134

15. Tahergorabi Z, Moodi M, Mesbahzadeh B. Breast cancer: a preventable disease. Journal of Birjand University of Medical Sciences. 2014;21(2):126-141. [Persian].

16. Nelson HD, Zakher B, Cantor A, et al. Risk factors for breast cancer for women aged 40 to 49 years: a systematic review and meta-analysis. Ann Intern Med. 2012;156(9):635-648. doi:10.7326/0003-4819-156-9-201205010-00006

17. Linos E, Willett WC, Cho E, Frazier L. Adolescent diet in relation to breast cancer risk among premenopausal women. Cancer Epidemiol Biomarkers Prev. 2010;19(3):689-696. doi:10.1158/1055-9965.epi-09-0802

(c) 2020 The Author (s); This is an open-access article distributed under the terms of the Creative Commons Attribution License (http://creativecommons.org/licenses/by/4.0), which permits unrestricted use, distribution, and reproduction in any medium, provided the original work is properly cited. 